\title{
Contributors to This Volume and Their Entries
}

Ainslie, George, Veterans' Administration Medical Center, Coatesville, PA, USA

Psychological Discount Rate

Allen, Michael W., Psychology, Victoria University of Wellington, P.O. Box 600 , Wellington, New Zealand

Poverty, Psychology of

Arnett, Dennis B., Marketing, Texas Tech University, P.O. Box 42101, Lubbock, TX 79409, USA

Philosophical-Methodological Foundations

Ball, Sheryl B., Economics, Virginia Polytechnic and State University, Blacksberg, VA 24061, USA

Experimental Economics

Beckmann, Suzanne C., Economics and Business Administration, Copenhagen Business School, Struenseegade 7-9, DK-2200 Copenhagen, Denmark

Ecology and Consumption; Emotions and Consumer Behaviour

Belk, Russell W., Marketing, David Eccles School of Business, Kendall D. Garff Building, University of Utah, Salt Lake City, UT 84112, USA Money

Berno, Tracy, Psychology, Human Sciences Division, P.O. Box 84, Lincoln University, Canterbury, New Zealand

Tourism, Psychology of

Berry, Christopher J., Politics, University of Glasgow, Glasgow G12 8QQ, Scotland, United Kingdom

Needs and Wants 
Bloch, Brian, International Business, University of Auckland, Private Bag 92019, Auckland 1, New Zealand

Speculation

Block, Lauren G., Marketing, Stern School of Business, New York University, New York, NY 10012, USA

Fear Appeals and Persuasion

Bolle, Friedel, Institut für Volkswirtschaftstheorie, Europa Universität Viadrina, Postfach 776, 15207 Frankfurt (Oder), Germany

Trust

Burgoyne, Carole B., Psychology, Washington Singer Laboratories, University of Exeter, Exeter EX4 4QG, United Kingdom

Gifts

Burt, Christopher D.B., Psychology, University of Canterbury, Private Bag 4800, Christchurch, New Zealand

Organizational Culture and Profitability

Burton, Scot, Marketing, College of Business Administration, University of Arkansas, Fayetteville, AR 72701, USA

Vanity

Caginalp, Gunduz, Mathematics, University of Pittsburgh, 507 Thackeray Hall, Pittsburgh, PA 15260, USA

Experimental Asset Markets

Cameron, Judy, Educational Psychology, 6-102 Education North, University of Alberta, Edmonton, Alberta, T6G 2G5, Canada

Rewards and the Myth of Performance Decrements

Choi, Young Back, Economics and Finance, St John's University, Jamaica, NY 11439, USA

Conventions

Cox, Anthony D., Marketing Indiana University School of Business, 801 West Michigan Street, Indianapolis, IN 46202, USA

Shoplifting 
Cox, Dena, Marketing Indiana University School of Business, 801 West Michigan Street, Indianapolis, IN 46202, USA

Shoplifting

Dabholkar, Pratibha A., Marketing, University of Tennessee, 307 Stokely Management Center, Knoxville, TN 37996, USA

Expectancy Value Models

Darity, William A. Jr, Economics, University of North Carolina at Chapel Hill, Chapel Hill, NC 27514, USA

Unemployment and Well-Being

Dawar, Niraj, Marketing, Richard Ivey School of Business, University of Western Ontario, London, Ontario N6A 3K7, Canada

Brand Equity; Perceived Quality

Dhar, Ravi, Marketing, Yale School of Management, Yale University, 135 Prospect Street, New Haven, CT 06511, USA

Choice Deferral

Dowling, Grahame R., Marketing, Australian Graduate School of Management, University of New South Wales, Sydney, NSW 2052, Australia Consumer Innovativeness; Perceived Risk

Duggan, Michael, Department of Psychology, Victoria University of Wellington, P.O. Box 600, Wellington, New Zealand

Share Markets and Psychology

Earl, Peter E., Economics, Commerce Division, P.O. Box 84, Lincoln University, Canterbury, New Zealand

Cognitive Dissonance; Personal Construct Theory

Ehrenberg, Andrew, Centre for Research in Marketing, South Bank Business School, South Bank University, London Road, London SE1 0AA, United Kingdom

Brand Loyalty

Elffers, Henk, Centre for Sociolegal Tax Research, Erasmus University, Rotterdam, P.O. Box 1738, 3000 DR Rotterdam, The Netherlands

Tax Evasion 
Endres, A.M., Economics, University of Auckland, Private Bag 92019, Auckland 1, New Zealand

Smith, Adam; Utility Theory

Fletcher, Garth, Psychology, University of Canterbury, Private Bag 4800, Christchurch, New Zealand

Attribution Theory

Ford, J.L., Economics, University of Birmingham, P.O. Box 363, Edgbaston, Birmingham B15 2TT, United Kingdom

Expectations

Foxall, Gordon R., Consumer Psychology, Cardiff Business School, Cardiff University, P.O. Box 920, Cardiff CF1 3XP, United Kingdom

Behaviourism

Friese, Susanne, Lehrstuhl für Konsumtheorie und Verbraucherpolitik (530/1) Universität Hohenheim, D-70593 Stuttgart, Germany

Addictive Buying

Friestad, Marian, Marketing, College of Business Administration, University of Oregon, Eugene, OR 97405, USA

Persuasion

Furnham, Adrian, Psychology, University College London, Gower Sreet, London WC1E 6BT, United Kingdom

Culture Shock

Gilleard, Christopher, Psychology, Springfield Hospital, Tooting SW17 7DJ, United Kingdom

McClelland Hypothesis

Goldsmith, Arthur H., Economics, Washington and Lee University, Lexington, VA 24450, USA

Unemployment and Well-Being

Gould, Stephen J., Marketing, Baruch College, The City University of New York, New York, NY 10010, USA

Introspective Research; Protocol and Cognitive Response Analysis 
Groenland, Edward A.G., Economic Psychology, Tilburg University, P.O. Box 90153, 5000 Le Tilburg, The Netherlands

Saving

Güith, Werner, Institut für Wirtschaftstheorie, Humboldt-Universität zu Berlin, Spandauer Str. 1, D-10178 Berlin, Germany

Game Theory

Hirschman, Elizabeth C., Marketing, School of Business, Janice H Levin Building, Livingston Campus, Rutgers University, Piscataway, NJ 08854, USA Hedonic Consumption

Hisrich, Robert D., Weatherhead School of Management, Case Western Reserve University, Enterprise Hall, 10900 Euclid Avenue, Cleveland, OH 44106, USA.

Entrepreneurship and Innovation

Holbrook, Morris B., Marketing, Graduate School of Business, 504 Uris Hall, Columbia University, New York, NY 10027, USA

Howard, John A.

Hudson, Stephen M., Psychology, University of Canterbury, Private Bag 4800, Christchurch, New Zealand

Addiction, Theories of

Hunt, Shelby D., Marketing, Texas Tech University, P.O. Box 42101, Lubbock, TX 79409, USA

Philosophical-Methodological Foundations

John, Deborah Roedder, Marketing, University of Minnesota, Minneapolis, MN 55455, USA

Children's Consumer Behaviour

Kagel, John H., Experimental Economics Laboratory, University of Pittsburgh, Pittsburgh, PA, USA

Animal Experiments in Economics

Kahneman, Daniel, Psychology, and the Woodrow Wilson School of Public and International Affairs, Princeton University, Princeton, NJ 08544, USA Heuristics and Biases 
Kemp, Simon, Psychology, University of Canterbury, Private Bag 4800, Christchurch, New Zealand

Inflation; Utility of Public Goods

Kirchler, Erich, Psychology, University of Vienna, Gölsdorfgasse 3/6, 1010 Vienna, Austria

Household Decision Making

Kleine, Susan Schultz, College of Business Administration, Bowling Green State University, Bowling Green, OH 43402, USA

Possessions

Klumb, Petra L., Abt für Gerontopsychiatrie, Freie Universitaet Berlin, Nussbaumallee 38, 14050 Berlin, Germany

Time Use

Koslow, Scott, Marketing, School of Management, University of Waikato, Private Bag 3105, Hamilton, New Zealand Conjoint Analysis

Laaksonen, Pirjo, Marketing, Faculty of Business Administration, University of Vaasa, PO Box 700, 65101 Vaasa, Finland Involvement

Langer, Ellen J., Psychology, Harvard University, William James Hall, 33 Kirkland Street, Cambridge, MA 02138, USA

Illusion of Control

Lavin, Marilyn, Marketing, School of Business and Economics, University of Wisconsin-Whitewater, Whitewater, WI 53190, USA

Soap Opera

Lea, Stephen E.G., Psychology, Washington Singer Laboratories, University of Exeter, Exeter EX4 4QG, United Kingdom

Credit, Debt and Problem Debt

Lewis, Alan, Economic Psychology, School of Humanities and Social Sciences, University of Bath, Claverton Down, Bath BA2 7AY, United Kingdom Morals, Markets and Green Investing 
Lichtenstein, Donald R., Marketing, College of Business Administration, University of Colorado, Boulder, CO 80309 , USA

Vanity

Lodewijks, John, Economics, University of New South Wales, Sydney, NSW 2052, Australia

Anthropology and Consumer Behaviour

Lutz, Mark A., Economics, University of Maine, 5774 Stevens Hall, Orono, ME 04469, USA

Dual Self; Humanistic Perspective

Lux, Kenneth, Clinical and Consulting Psychologist, Steven Mills Professional Building, 1441 Hotel Road, Auburn, ME 04210, USA

Dual Self

Maani, Sholeh A., Economics, University of Auckland, Private Bag 92019, Auckland 1, New Zealand

Labour Supply; Work Effort

MacFadyen, Alan J., Economics, University of Calgary, 2500 University Drive NW, Calgary, Alberta T2N 1NS, Canada.

Well-Being

Mayer, Robert N., Family and Consumer Studies, University of Utah, Salt Lake City, UT 84112, USA

Consumer Protection

Mengüç, Bülent, Marketing, Commerce Division, P.O. Box 84, Lincoln University, Canterbury, New Zealand

Search Processes

Miller, Christopher M., Marketing, Thunderbird: The American Graduate School of International Management, 15249 N. 59th Ave, Glendale, AZ 85306, USA

Fashion

Mollenkopf, Diane, Marketing, Commerce Division, P.O. Box 84, Lincoln University, Canterbury, New Zealand

Congruity Theory 
Moore, Kevin, Psychology, Human Sciences Division, P.O. Box 84, Lincoln University, Canterbury, New Zealand

Leisure, Psychology of

Moore, Vanessa, Marketing, Commerce Division, P.O. Box 84, Lincoln University, Canterbury, New Zealand

Congruity Theory

Muthoo, Abhinay, Economics, University of Essex, Wivenhoe Park, Colchester, CO4 3SQ, United Kingdom

Rationality in the Face of Uncertainty

Nelson, Paul, Marketing, William E Simon Graduate School of Business Administration, University of Rochester, Rochester, NY, USA

Multiattribute Utility Models; Positioning

Netemeyer, Richard G., Marketing, E.J. Ourso College of Business, Louisiana State University, Baton Rouge, LA 70803, USA

Vanity

Ng, Sik Hung, Psychology, Victoria University of Wellington, P.O. Box 600, Wellington, New Zealand

Poverty, Psychology of

O'Curry, Suzanne, Economics, College of Business, DePaul University, 1 E. Jackson Boulevard, Chicago, IL 60604, USA

Budgeting and Mental Accounting

OlekaIns, Mara, Management and Industrial Relations, University of Melbourne, Parkville, Victoria 3052, Australia

Negotiation

Ozanne, Julie L., Marketing, R.B. Pamplin College of Business, Virginia Polytechnic and State University, Blacksberg, VA 24061, USA

Hermeneutics

Peñaloza, Lisa, Marketing, College of Business, University of Colorado, Campus Box 419, Boulder, CO 80309-0419, USA Acculturation 
Phelps, Charlotte, D., Economics, School of Business and Management, Temple University, Philadelphia, PA 19122, USA

Altruism

Porter, David, Humanities and Social Sciences, California Institute of Technology, Pasadena, CA 91125, USA

Experimental Asset Markets

Quiggin, John, Economics, The Faculties, Australian National University, ACT 0200, Australia

Utility

Rajendran, K.N., Marketing, University of Northern Iowa, Cedar Falls, IA 50614, USA

Reference Price

Richins, Marsha L., Marketing, College of Business and Public Administration, University of Missouri, Columbia, MO 65211, USA Material Values

Roland-Lévy, Christine, Laboratoire de Psychologie Sociale, Université Rene Descartes Paris V, 28 Rue Serpente, F-75270 Paris Cedex 06, France Economic Socialization

Rook, Dennis W., Marketing, Marshall School of Business, University of Southern California, Los Angeles, CA 90089, USA

Impulse Buying; Ritual

Routh, David A., Psychology, University of Bristol, 8 Woodland Rd., Bristol BS8 1TN, United Kingdom

Lay Economic Beliefs

Schoormans, Jan, Consumer Research, Faculty of Industrial Design, Jaffalaan 9,2628 BX Delft, The Netherlands

Consumer Knowledge

Scott, Linda M., Advertising, University of Illinois at Urbana-Champaign, Urbana, IL 61801, USA

Images in Advertising; Women in Advertising 
Scriven, John, Centre for Research in Marketing, South Bank Business School, South Bank University, London Road, London SE1 OAA, United Kingdom

Brand Loyalty

Sent, Esther-Mirjam, Economics, 426 Decio Hall, University of Notre Dame, Notre Dame, IN 46556, USA

Satisficing

Shafir, Eldar, Psychology, and the Woodrow Wilson School of Public and International Affairs, Princeton University, Princeton, NJ 08544, USA Heuristics and Biases

Singer, Alan E., Management, University of Canterbury, Private Bag 4800, Christchurch, New Zealand

Escalation of Commitment; Rationality, General Theory of

Singer, Ming, Psychology, University of Canterbury, Private Bag 4800, Christchurch, New Zealand

Faimess

Smith, Vernon L., Economic Science Laboratory, Karl Eller Graduate School of Management, University of Arizona, Tucson, AZ 85721, USA

Experimental Asset Markets

Spash, Clive L., Department of Land Economy, University of Cambridge, 19 Silver Street, Cambridge, CB3 9EP, United KIngdom

Contingent Valuation

Stacey, Barrie G., Lincoln Gerontology Centre, LaTrobe University, Bundoora, Victoria 3083, Australia

Growing Old

Stern, Barbara B., Marketing, Ackerson Hall, Newark Campus, Rutgers University, Newark, NJ 07102, USA

Literary Explication and Deconstruction

Sujan, Mita, Marketing, Smeal College of Business Administration Pennsylvania State University, University Park, Pittsburgh, PA 16802, USA Bettman, James $R$. 
Tay, Richard S., Economics, Commerce Division, P.O. Box 84, Lincoln University, Canterbury, New Zealand

Discrete Choice Models

Trivedi, Minakshi, Marketing, State University of New York at Buffalo, Jacobs Management Center, PO Box 604000, Buffalo, NY 14260, USA Variety-Seeking Behaviour

Troilo, Gabriele, Marketing, SDA Bocconi (Business School of Bocconi University) via Bocconi 8, 20136 Milan, Italy

Collecting

Twomey, Paul J., Economics, King's College, Cambridge, United Kingdom Habit

Tyszka, Tadeusz, Psychology, Polish Academy of Sciences, Podlesna 61, PL 00-967 Warsaw, Poland

Transformation in Eastern Europe

Vandekerckhove, Marie M.P., Ableilung Psychologie, Universität Bielefeld, 33501 Bielefeld, Germany

Conspicuous Consumption

Van Raaij, W. Fred, Economics and Psychology, Erasmus University, P.O. Box 1738, 3000 DR Rotterdam, The Netherlands

History of Economic Psychology

Venkatesh, Alladi, Marketing, Graduate School of Management, University of California at Irvine, Irvine, CA 92717, USA

Postmodernism and Consumption

Veum, Jonathan R., Research Economist, Bureau of Labor Statistics, Room 4945, 2 Massachusetts Avenue, NE, Washington, DC 20212, USA

Unemployment and Well-Being

Walker, Michael, Psychology, University of Sydney, Sydney, NSW 2006, Australia

Gambling 
Ward, Colleen A., Social Work and Psychology, National University of Singapore, Kent Ridge, Singapore, 119260

Cross-Cultural Research

Ward, Tony, Psychology, University of Canterbury, Private Bag 4800, Christchurch, New Zealand

Addiction, Theories of

Wärneryd, Karl-Erik, Stockholm School of Economics, P.O. Box 6501, 11383 Stockholm, Sweden.

Katona, George

Webley, Paul, Psychology, Washington Singer Laboratories, University of Exeter, Exeter EX4 4QG, United Kingdom

Children's Saving

White, Judith B., Psychology, Harvard University, William James Hall, 33 Kirkland Street, Cambridge, MA 02138, USA

Illusion of Control

Wicklund, Robert A., Ableilung Psychologie, Universität Bielefeld, 33501 Bielefeld, Germany

Cognitive Dissonance; Conspicuous Consumption

Wilkes, Robert E., Marketing, College of Business Administration, Texas Tech University, Lubbock, TX 79049, USA

Household Life Cycle 